\title{
On Coverage of 3D Terrains by Wireless Sensor Networks
}

\author{
Mostefa Zafer \\ Ecole nationale Supérieure d'Informatique, \\ BP 68M, 16309, Oued-Smar, Alger, Algérie, \\ Email: m_zafer@esi.dz
}

\author{
Mustapha Reda Senouci, Mohamed Aissani \\ Ecole Militaire Polytechnique, \\ BP 17, 16046, Bordj El-Bahri, Alger, Algérie, \\ Email: \{mrsenouci, maissani\}@gmail.com
}

\begin{abstract}
The coverage of a Region of Interest (RoI), that must be satisfied when deploying a Wireless Sensor Network (WSN), depends on several factors related not only to the sensor nodes (SNs) capabilities but also to the RoI topography. This latter has been omitted by most previous deployment approaches, which assume that the RoI is 2D. However, some recent WSNs deployment approaches dropped this unrealistic assumption. This paper surveys the different models adopted by the state-of-theart deployment approaches. The weaknesses that need to be addressed are identified and some proposals expected to enhance the practicality of these models are discussed.
\end{abstract}

\section{INTRODUCTION}

$\mathbf{T}$ HE WSN deployment on 3D RoI presents many difficulties evoked by the topography, which has a direct impact on the coverage quality. Indeed, the presence of obstacles can hinder the detection of the target event. Also, the use of mobile SNs to eliminate the coverage voids becomes increasingly difficult, and the random deployment generates a very low coverage quality for 3D terrains [1]. Thus, ensuring the coverage of a 3D RoI by a WSN requires a deterministic deployment of the SNs, which consists in precomputing their number and their positions [2]. The resolution of this NPhard problem [3] goes through a formulation phase, which describes the impact of the different factors on the coverage quality, and provides in its end an expression measuring the coverage quality produced by a given deployment scheme.

Once formulated, the problem is solved using heuristics or meta-heuristics, to select an appropriate deployment scheme. The practicality of a selected solution depends on the formulation process, which has been accomplished differently in the literature, depending on the factors taken into account and the modeling of their impacts on the coverage quality. When deploying WSNs on 3D RoIs, the formulation phase needs to be enriched by adding the RoI topography factor. To do this, it is necessary as a first step to model the SNs sensing capability, taking into account the RoI impact. In the second step, this model is used to formulate the RoI coverage. In this paper, we survey existing formulations of the coverage of $3 \mathrm{D}$ RoIs by WSNs in order to identify their main shortcomings that must be eliminated and make them more realistic. The coverage models and the RoI coverage deduction are detailed in Sections II and III, respectively. Section IV discusses the reliability of these models and some open issues. Section V concludes the paper.

\section{Coverage Models}

To estimate the coverage quality of the RoI, produced by the deployment of a WSN composed of $\mathcal{N}$ SNs, it is necessary to check the coverage status of each point $p_{i}$ of the RoI. This status is deduced from a basic information $\mathcal{C}\left(p_{i}, s_{j}\right)$, which is the state of coverage of $p_{i}$ by each $\mathrm{SN} s_{j}$. The factors considered in estimating $\mathcal{C}\left(p_{i}, s_{j}\right)$, and the formulation of their impacts on $\mathcal{C}\left(p_{i}, s_{j}\right)$ represent the coverage model [4]. In the existing coverage models, $\mathcal{C}\left(p_{i}, s_{j}\right)$ is formulated according to one or more of the following factors: (i) the sensing range of $s_{j}$; (ii) the sensing angle of $s_{j}$; (iii) the topography of the RoI; (iv) the weather permeability of the RoI, and (v) the permeability of the objects separating $p_{i}$ and $s_{j}$. It should be noted that the first and second factors are part of the SNs characteristics, while the third, fourth, and fifth factors belong to the RoI characteristics. Consequently, the most general formula of $\mathcal{C}\left(p_{i}, s_{j}\right)$ is given by Eq. 1, where $\mu_{d}\left(p_{i}, s_{j}\right), \mu_{\phi}\left(p_{i}, s_{j}\right), \mu_{v}\left(p_{i}, s_{j}\right), \mu_{w}\left(p_{i}, s_{j}\right)$, and $\mu_{o}\left(p_{i}, s_{j}\right)$ are binary or probabilistic functions, modeling the impact of the first, second, third, fourth, and fifth factors, respectively. In the sequel, we discuss the different models proposed to consider the impact of these factors on $\mathcal{C}\left(p_{i}, s_{j}\right)$.

$$
\mathcal{C}\left(p_{i}, s_{j}\right)=\mu_{d}\left(p_{i}, s_{j}\right) \times \mu_{\phi}\left(p_{i}, s_{j}\right) \times \mu_{v}\left(p_{i}, s_{j}\right) \times \mu_{w}\left(p_{i}, s_{j}\right) \times \mu_{o}\left(p_{i}, s_{j}\right)
$$

\section{A. Impact of the SNs sensing range}

The sensing range of $s_{j}$ is a reference distance $r_{s}$ from which we can pronounce on the coverage of $p_{i}$ by $s_{j}$ in function of their distance $d\left(p_{i}, s_{j}\right)$ [5], [6], [7], [8]. The influence of this factor on $\mathcal{C}\left(p_{i}, s_{j}\right)$, modeled by the function $\mu_{d}\left(p_{i}, s_{j}\right)$, takes three forms: (i) Deterministic impact [5], [6], where $\mathcal{C}\left(p_{i}, s_{j}\right)$ is constant with respect to $d\left(p_{i}, s_{j}\right)$, as long as $p_{i}$ is in the sensing range of $s_{j}$. Otherwise, $\mathcal{C}\left(p_{i}, s_{j}\right)$ is null; (ii) Probabilistic impact [9], [10], [7], where $\mathcal{C}\left(p_{i}, s_{j}\right)$ degrades with respect to $d\left(p_{i}, s_{j}\right)$, and it becomes null when the point $p_{i}$ is outside the sensing range of $s_{j}$; (iii) Hybrid impact [11], [12], [13], by considering that $s_{j}$ has two sensing ranges, the first is "with certitude", noted $r_{1}$, and the second is "without certitude", noted $r_{2}$, where $r_{2}>r_{1}$. Thus, $\mathcal{C}\left(p_{i}, s_{j}\right)$ is constant with respect to $d\left(p_{i}, s_{j}\right)$, as long as $p_{i}$ is in the sensing range "with certitude" of $s_{j}$; it is null when $p_{i}$ is outside the sensing range "without certitude" of $s_{j}$, and it degrades with 
respect to $d\left(p_{i}, s_{j}\right)$, in the remaining case. Fig. 1 shows 2Dgraphical representations of these models.

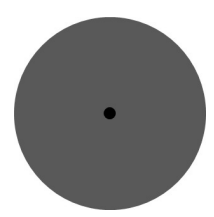

(a) Binary

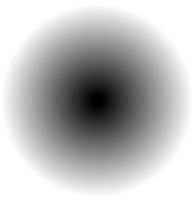

(b) Probabilistic

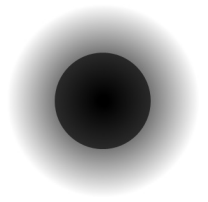

(c) Hybrid
Fig. 1. Impact of the sensing range.

\section{B. Impact of the SNs sensing angle}

Sometimes, the sensing capability of SNs is limited to an angle $\phi$. In this case, the coverage of $p_{i}$ by $s_{j}$ depends on the position of $p_{i}$ with respect to the orientation of $s_{j}$, represented by the unit vector $\overrightarrow{u_{s}}$. Thus, the position of $p_{i}$ with respect to the orientation of $s_{j}$, is measured by the angle $\left(\overrightarrow{u_{s}}, \overrightarrow{s_{j} p_{i}}\right)$, whose impact on $\mathcal{C}\left(p_{i}, s_{j}\right)$ takes three forms: (i) Deterministic impact [14], [6], [15], which means that $\mathcal{C}\left(p_{i}, s_{j}\right)$ is constant with respect to $\left(\overrightarrow{u_{s}}, \overrightarrow{s_{j} p_{i}}\right)$, as long as $p_{i}$ is in the sensing angle of $s_{j}$. Otherwise, $\mathcal{C}\left(p_{i}, s_{j}\right)$ is null; (ii) Probabilistic impact [9], [10], which means that $\mathcal{C}\left(p_{i}, s_{j}\right)$ degrades in function of $\left(\overrightarrow{u_{s}}, \overrightarrow{s_{j} p_{i}}\right)$, and it becomes null when $p_{i}$ is outside the sensing angle of $s_{j}$; (iii) Hybrid impact [16], [17], which means that $s_{j}$ has two sensing angles, the first is "with certitude", noted $\phi_{1}$, and the second is "without certitude", noted $\phi_{2}$, where $\phi_{2}>\phi_{1}$. Thus, $\mathcal{C}\left(p_{i}, s_{j}\right)$ is constant with respect to $\left(\overrightarrow{u_{s}}, \overrightarrow{s_{j} p_{i}}\right)$, as long as $p_{i}$ is in the sensing angle "with certitude" of $s_{j}$; it is null when $p_{i}$ is not in the sensing angle "without certitude" of $s_{j}$; and it degrades depending on $\left(\overrightarrow{u_{s}}, \overrightarrow{s_{j} p_{i}}\right)$, in the remaining case. Fig. 2 shows $2 \mathrm{D}$-graphical representations of these models.

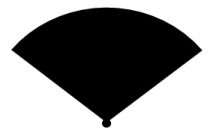

(a) Binary

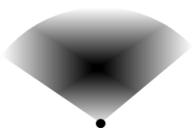

(b) Probabilistic

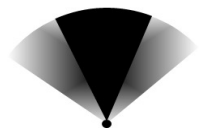

(c) Hybrid
Fig. 2. Impact of the sensing angle.

\section{Impact of the RoI topography}

To take into account the topography impact on $\mathcal{C}\left(p_{i}, s_{j}\right)$, most formulations proceed to verify the "visibility" between $p_{i}$ and $s_{j}$, by using the Line of Sight (LoS) [15], [18], [19] method (Fig. 3). This latter selects $N_{v}$ points $q_{i}\left(x_{i}, y_{i}, z_{i}\right)_{1 \leq i \leq N_{v}}$, located on the segment $\left[p_{i} s_{j}\right.$, and compares the altitude $z_{i}$ of each point $q_{i}$ with the RoI height $\mathcal{E}\left(x_{i}, y_{i}\right)$, provided by a terrain model $\mathcal{E}$. Thus, $p_{i}$ and $s_{j}$ are considered inter-visible, if each point $q_{i}$ is above the terrain. Once the visibility between $p_{i}$ and $s_{j}$ verified, its impact on $\mathcal{C}\left(p_{i}, s_{j}\right)$ takes two forms: (i) Deterministic impact [20], [15], [18], [19], by considering that $p_{i}$ can be covered by $s_{j}$, only if $p_{i}$ and $s_{j}$ are inter-visible. In the opposite case,
$\mathcal{C}\left(p_{i}, s_{j}\right)$ is null. (ii) Probabilistic impact [14] by considering that $\mathcal{C}\left(p_{i}, s_{j}\right)$ deteriorates (does not cancel out) according to the number of obstacles separating $p_{i}$ and $s_{j}$. Some WSNs deployment approaches on 3D terrains do not consider the visibility factor when formulating the coverage. This choice is based on one of the following justifications. (i) Some events are detectable even if they occur in locations invisible to the SNs [21]; (ii) The terrain is assumed to be sufficiently convex, so that the visibility between a $\mathrm{SN}$ and any point within its sensing range is always possible [3], [1], [22]; (iii) The impact of the RoI topography, is already taken into account during the parameterization of $\mathcal{C}\left(p_{i}, s_{j}\right)$ depending on $d\left(p_{i}, s_{j}\right)$ [6], [7].

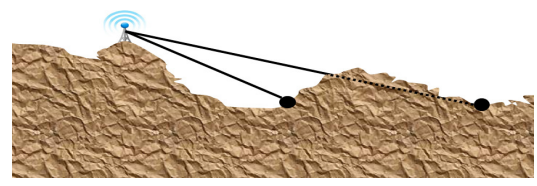

Fig. 3. LoS concept.

\section{Impact of the RoI weather and the RoI objects}

In [23], $\mathcal{C}\left(p_{i}, s_{j}\right)$ is considered dependent on the permeability of the objects and the weather of the RoI, where their impacts on $\mathcal{C}\left(p_{i}, s_{j}\right)$ is formulated separately in a probabilistic way.

On Table I, we list the different coverage models, denoted $C_{1}, C_{2}, \cdots, C_{12}$, adopted by the WSNs deployment approaches on $3 \mathrm{D}$ surfaces, where the difference between them lies in the factors taken into account and how their influences on $\mathcal{C}\left(p_{i}, s_{j}\right)$ is modeled. The type of each model, which can be binary or probabilistic, is determined by the possible values of $\mathcal{C}\left(p_{i}, s_{j}\right)$. Indeed, a coverage model is binary if $\mathcal{C}\left(p_{i}, s_{j}\right) \in\{0,1\}$, and it is considered probabilistic if $\mathcal{C}\left(p_{i}, s_{j}\right) \in[0,1]$.

\section{RoI COVERAGE}

The basic information $\mathcal{C}\left(p_{i}, s_{j}\right)$ is used to formulate the coverage state $\operatorname{Cov}\left(p_{i}, \mathcal{N}\right)$ of each point $p_{i}$ with respect to the WSN composed of $\mathcal{N}$ SNs. If the adopted coverage model is binary [6], $p_{i}$ can be in two states with respect to the WSN: " $p_{i}$ is covered", if it is covered by at least one $\mathrm{SN}$, and " $p_{i}$ is not covered", if it is not covered by any SN. Therefore, $\operatorname{Cov}\left(p_{i}, \mathcal{N}\right)=\max _{1 \leq j \leq \mathcal{N}} \mathcal{C}\left(p_{i}, s_{j}\right)$. If the adopted coverage model is probabilistic, and $\mathcal{C}\left(p_{i}, s_{j}\right)$ is interpreted as the probability of coverage of $p_{i}$ by $s_{j}[14], \mathcal{C o v}\left(p_{i}, \mathcal{N}\right)$ is given by $\operatorname{Cov}\left(p_{i}, S\right)=1-\prod_{1 \leq j \leq \mathcal{N}}\left(1-\mathcal{C}\left(p_{i}, s_{j}\right)\right)$. If the adopted coverage model is probabilistic and $\mathcal{C}\left(p_{i}, s_{j}\right)$ is interpreted as the coverage quality of $p_{i}$ by $s_{j}$ [23], [11], [12], $\operatorname{Cov}\left(p_{i}, \mathcal{N}\right)$ is equal to $\max _{1 \leq j \leq \mathcal{N}} \mathcal{C}\left(p_{i}, s_{j}\right)$, which means that the coverage of $p_{i}$ is assigned to the $\mathrm{SN}$ that offers the best coverage quality for $p_{i}$. The last step in the formulating phase is to express the coverage quality $\operatorname{Cov}(\mathcal{A}, \mathcal{N})$ of the $\operatorname{RoI} \mathcal{A}$ by the WSN composed of $\mathcal{N}$ SNs, using the state $\operatorname{Cov}\left(p_{i}, \mathcal{N}\right)$ of each point $p_{i} \in \mathcal{A}$. This step is strongly related to the terrain model $\mathcal{E}$ adopted to represent $\mathcal{A}$ (Fig. 4), which may 
TABLE I

VARIOUS COVERAGE MODELS USED IN THE LITERATURE.

\begin{tabular}{|c|c|c|c|c|c|c|c|}
\hline \multirow{3}{*}{ Model } & \multirow{3}{*}{ Type } & \multicolumn{5}{|c|}{ Impact of considered factors } & \multirow{3}{*}{ References } \\
\hline & & \multicolumn{2}{|c|}{ SNs characteristics } & \multicolumn{3}{|c|}{ RoI characteristics } & \\
\hline & & Detection range & Detection angle & Topography & Weather & Object & \\
\hline$C_{1}$ & Binary & Binary & . & . & . & . & {$[3],[1],[5],[8],[24],[25]$} \\
\hline$C_{2}$ & Binary & Binary & . & Binary & . & . & {$[26],[20],[18],[19],[4],[27$} \\
\hline$C_{3}$ & Probabilistic & Probabilistic & . & . & . & . & {$[22],[7],[28]$} \\
\hline$C_{4}$ & Probabilistic & Hybrid & . & . & . & . & [21] \\
\hline$C_{5}$ & Probabilistic & Hybrid & . & Binary & . & . & {$[11],[12],[13],[29]$} \\
\hline$C_{6}$ & Probabilistic & Hybrid & Hybrid & Binary & . & . & [16], [17], [30] \\
\hline$C_{7}$ & Binary & Binary & Binary & . & . & . & {$[6]$} \\
\hline$C_{8}$ & Binary & Binary & Binary & Binary & . & . & [15], [31] \\
\hline$C_{9}$ & Probabilistic & Binary & Binary & Probabilistic & . & . & [14] \\
\hline$C_{10}$ & Probabilistic & Probabilistic & Probabilistic & Binary & . & . & [9], [10] \\
\hline$C_{11}$ & Probabilistic & Binary & Binary & Binary & Probabilistic & Probabilistic & [23] \\
\hline$C_{12}$ & Probabilistic & Hybrid & Probabilistic & Binary & . & . & [32] \\
\hline
\end{tabular}

be discontinuous, such as the matrix model or continuous, such as the mathematical and the TIN (Triangulated Irregular Network) models.

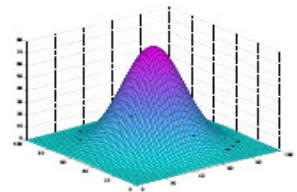

(a) Mathematical

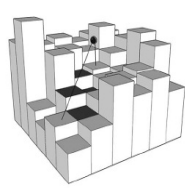

(b) Matrix

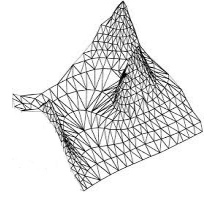

(c) $\mathrm{TIN}$
Fig. 4. Various terrain models used in the literature.

In the matrix model, $\operatorname{Cov}(\mathcal{A}, \mathcal{N})$ represents the rate of points of $\mathcal{E}$ covered by the $\mathcal{N}$ SNs [11], [12], [15], [18], considering that these points have the same level [11], [12], [13], [15], [18] or different levels [10] of importance. Thus, $\operatorname{Cov}(\mathcal{A}, \mathcal{N})$ is given by Eq. 2, where the weight $w_{i}$ represents the importance assigned to a point $p_{i} \in \mathcal{E}$.

$$
\operatorname{Cov}(\mathcal{A}, \mathcal{N})=\frac{\sum_{p_{i} \in \mathcal{E}} w_{i} \cdot \mathcal{C} \operatorname{ov}\left(p_{i}, \mathcal{N}\right)}{\sum_{p_{i} \in \mathcal{E}} w_{i}}
$$

Most approaches based on the mathematical model [5], [28] construct a matrix model $\dot{\mathcal{E}}$ from the original model $\mathcal{E}$. Hence, $\operatorname{Cov}(\mathcal{A}, \mathcal{N})$ is computed according to Eq. 2, considering only the points of $\dot{\mathcal{E}}$. This transformation is avoided in some approaches [6]. The alternative idea is to estimate, using a geometric calculation, the surface $\left\|\mathcal{A}_{j}\right\|$ of the portion $\mathcal{A}_{j} \subset \mathcal{A}$ covered by each $\mathrm{SN} s_{j}$. Thus, $\operatorname{Cov}(\mathcal{A}, \mathcal{N})$ is computed as the ratio of the surface covered by the SNs and the surface $\|\mathcal{A}\|$ of $\mathcal{A}$ (Eq. 3).

$$
\operatorname{Cov}(\mathcal{A}, \mathcal{N})=\frac{\left\|\bigcup_{1 \leq j \leq \mathcal{N}} \mathcal{A}_{j}\right\|}{\|\mathcal{A}\|}
$$

The approaches based on the TIN model formulate firstly the coverage quality $\operatorname{Cov}\left(t_{i}, \mathcal{N}\right)$ of each triangle $t_{i} \in \mathcal{E}$ [3], [1], [22], [23], which is calculated as the average of the coverage quality of the important points of $t_{i}$ (its center of gravity and its vertexes). After that, $\operatorname{Cov}(\mathcal{A}, \mathcal{N})$ is computed as the average of the coverage qualities of all the triangles $t_{i} \in \mathcal{E}$, assuming that these triangles have the same level of importance [4] or different levels [23]. The formula of $\operatorname{Cov}(\mathcal{A}, \mathcal{N})$ is given by Eq. 4 , where $w_{i}$ represent the importance affected to the triangle $t_{i} \in \mathcal{E}$.

$$
\mathcal{C o v}(\mathcal{A}, \mathcal{N})=\frac{\sum_{t_{i} \in \mathcal{E}} w_{i} \cdot \operatorname{Cov}\left(t_{i}, \mathcal{N}\right)}{\sum_{t_{i} \in \mathcal{E}} w_{i}}
$$

\section{Discussions}

The first important remark concerns the choice of the terrain model to represent the RoI. This choice is based, in most cases, on criteria other than reliability. For instance, the matrix model was used in [14], [9], although the TIN model is more appropriate. Additionally, the assumptions adopted in some formulations [3], [1] to justify the omission of the visibility factor are unfounded. Indeed, the assumption that the RoI is sufficiently smooth, where the visibility between a $\mathrm{SN}$ and all the points in its sensing range is guaranteed, is not realistic. Moreover, in the case where the visibility factor is considered [23], [14], its impact is formulated independently of the nature of the phenomenon being monitored and the type of SNs (laser, radio, etc.) used to detect it. In fact, these two parameters determine the manner (probabilistic or deterministic) and the degree of visibility impact on the coverage quality.

Moreover, some formulations adopt clearly unrealistic assumptions, such as the deterministic impact of the various factors on the coverage quality [3], [1], as well as the omnidirectional sensing capability of the SNs [22], [21]. Furthermore, most of the formulations [5], [6] do not consider the constraints imposed by the RoI, which limits the possible positions of the SNs. The existence of such constraints in complex 3D terrains is very likely. As a result, we believe that the design of a realistic coverage model remains an open issue. Its resolution requires to consider not only the type of SNs to be used but also the phenomenon to be monitored as well 
as experimental tests, allowing the correct parameterization of their impacts and the deduction of the real relationship between them.

\section{CONCLUSION}

Examining the several formulations of 3D terrain coverage by WSNs allowed us to confirm that this paramount process remains an open issue. We believe that carrying out experimental tests, to correctly assess and model both the influence of the above-discussed factors and the real relationship between them is the first step towards a practical and effective solution. As a future work, we plan to carry out an in-depth evaluation of existing resolution approaches related to the problem of $3 \mathrm{D}$ terrains coverage by WSNs. This will allow us to gain a better understanding in order to provide additional guidelines on the appropriate choice of the modeling/resolution approaches.

\section{REFERENCES}

[1] L. Kong, M.-C. Zhao, X.-Y. Liu, J. Lu, Y. Liu, M.-Y. Wu, and W. Shu, "Surface Coverage in Sensor Networks," TPDS, vol. 25, no. 1, 2014 doi: 10.1109/TPDS.2013.35

[2] M. R. Senouci and A. Mellouk, Deploying Wireless Sensor Networks: Theory and Practice. Elsevier, 2016. ISBN 978-1-78548-099-7

[3] M.-C. Zhao, J. Lei, M.-Y. Wu, Y. Liu, and W. Shu, "Surface Coverage in Wireless Sensor Networks," in INFOCOM, 2009. doi: 10.1109/INFCOM.2009.5061912

[4] M. Zafer, M. R. Senouci, and M. Aissani, "Terrain Partitioning Based Approach for Realistic Deployment of Wireless Sensor Networks," in CIIA'18, 2018. doi: 10.1007/978-3-319-89743-13_7

[5] K. Kim, "Mountainous terrain coverage in mobile sensor networks," IET Comm., vol. 9, no. 5, 2015. doi: 10.1049/iet-com.2014.0443

[6] F. Xiao, X. Yang, M. Yang, L. Sun, R. Wang, and P. Yang, "Surface Coverage Algorithm in Directional Sensor networks for ThreeDimensional Complex Terrains," TST, vol. 21, no. 4, 2016. doi: 10.1109/TST.2016.7536717

[7] T. Song, C. Gong, and C. Liu, "A practical coverage algorithm for wireless sensor networks in real terrain surface," IJWMC, vol. 5, no. 4 2012. doi: 10.1504/IJWMC.2012.051514

[8] F. Li, J. Luo, W. Wang, and Y. He, "Autonomous Deployment for Load Balancing k-Surface Coverage in Sensor Networks," TWC, vol. 14, no. 1, 2015. doi: 10.1109/TWC.2014.2341585

[9] V. Akbarzadeh, C. Gagné, M. Parizeau, and M. A. Mostafavi, "Black-box Optimization of Sensor Placement with Elevation Maps and Probabilistic Sensing Models," in Int. Symp., 2011. doi 10.1109/ROSE.2011.6058544

[10] V. Akbarzadeh, C. Gagné, M. Parizeau, M. Argany, and M. A. Mostafavi, "Probabilistic Sensing Model for Sensor Placement Optimization Based on Line-of-Sight Coverage," ToIM, vol. 62, no. 2, 2013. doi: 10.1109/TIM.2012.2214952

[11] N. Unaldi, S. Temel, and V. K. Asari, "Method for Optimal Sensor Deployment on 3D Terrains Utilizing a Steady State Genetic Algorithm with a Guided Walk Mutation Operator Based on the Wavelet Transform," Sensors, vol. 12, no. 4, 2012. doi: 10.3390/s120405116

[12] S. Temel, N. Unaldi, and O. Kaynak, "On Deployment of Wireless Sensors on 3D Terrains to Maximize Sensing Coverage by Utilizing Cat Swarm Optimization with Wavelet Transform," TSMC, vol. 44, no. 1, 2014. doi: 10.1109/TSMCC.2013.2258336

[13] Y. Hang, L. Xunbo, W. Zhenlin, Y. Wenjie, and H. Bo, "A Novel Sensor Deployment Method Based on Image Processing and Wavelet Transform to Optimize the Surface Coverage in WSNs," CJE, vol. 25, no. 3, 2016 doi: $10.1049 /$ cje.2016.05.015
[14] N. T. Tam, H. D. Thanh, L. H. Son, and V. T. Le, "Optimization for the sensor placement problem in 3D environments," in ICNSC, 2015. doi: 10.1109/ICNSC. 2015.7116057

[15] V. Akbarzadeh, A. H.-R. Ko, C. Gagné, and M. Parizeau, "TopographyAware Sensor Deployment Optimization with CMA-ES," in ICPPSN, 2010. doi: 10.1007/978-3-642-15871-1_15

[16] B. Cao, J. Zhao, Z. Lv, and X. Liu, "3D Terrain Multiobjective Deployment Optimization of Heterogeneous Directional Sensor Networks in Security Monitoring," TBD, vol. 14, no. 8, 2015. doi: 10.1109/TBDATA.2017.2685581

[17] B. Cao, J. Zhao, Z. Lv, X. Liu, X. Kang, and S. Yang, "Deployment Optimization for 3D Industrial Wireless Sensor Networks Based on Particle Swarm Optimizers with Distributed Parallelism,” JNCA, 2017. doi: 10.1016/j.jnca.2017.08.009

[18] S. Doodmana, A. Afghantoloee, M. A. Mostafavi, and F. Karimipour, "3D extention of the VOR algorithm to determine and optimize the coverage of geosensor networks," in ISPRS, 2014. doi: 10.5194/isprsarchives-XL-2-W3-103-2014

[19] A. H.-R. Ko and F. Gagnon, "Process of 3D wireless decentralized sensor deployment using parsing crossover scheme," EACI, vol. 11, 2015. doi: 10.1016/j.aci.2014.11.001

[20] K. Veenstra and K. Obraczka, "Guiding Sensor Node Deployment Over 2.5D Terrain,” in ICC, 2015. doi: 10.1109/ICC.2015.7249396

[21] J.-H. Seo, Y. Yoon, and Y.-H. Kim, "An Efficient Large-Scale Sensor Deployment Using a Parallel Genetic Algorithm Based on CUDA," IJDSN, vol. 2016, 2015. doi: 10.1155/2016/8612128

[22] M. Jin, G. Rong, H. Wu, L. Shuai, and X. Guo, "Optimal Surface Deployment Problem in Wireless Sensor Networks," in INFOCOM, 2012. doi: 10.1109/INFCOM.2012.6195622

[23] H. R. Topcuoglu, M. Ermis, and M. Sifyan, "Positioning and Utilizing Sensors on a 3D Terrain Part I: Theory and Modeling," TSMC, vol. 41, no. 3, 2011. doi: 10.1109/TSMCC.2010.2055850

[24] N. Boufares, I. Khoufi, P. Minet, and L. Saidane, "Covering a 3D flat surface with autonomous and mobile wireless sensor nodes," in PEMWN 2017. doi: 10.1109/IWCMC.2017.7986528

[25] C. Wang and H. Jiang, "SURF: A Connectivity-based Space Filling Curve Construction Algorithm in High Genus 3D Surface WSNs," in CCC, 2015. doi: 10.1109/INFOCOM.2015.7218470

[26] A. T. Murray, K. Kim, J. W. Davis, R. Machiraju, and R. Parent, "Coverage optimization to support security monitoring," CEUS, vol. 31, 2007. doi: 10.1016/j.compenvurbsys.2006.06.002

[27] B. Cao, J. Zhao, P. Yang, Z. Lv, X. Liu, X. Kang, S. Yang, K. Kang, and A. Anvari-Moghaddam, "Distributed parallel cooperative coevolutionary multi-objective large-scale immune algorithm for deployment of wireless sensor networks," FGCS, vol. 82, 2018. doi: 10.1016/j.future.2017.10.015

[28] L. Feng, Z. Sun, and T. Qiu, "Genetic Algorithm-Based 3D Coverage Research in Wireless Sensor Networks," in ICCISIS, 2013. doi: 10.1109/CISIS.2013.112

[29] N. Unaldi and S. Temel, "Wireless Sensor Deployment Method on 3D Environments to Maximize Quality of Coverage and Quality of Network Connectivity," in WCECS, 2014, pp. 1-6.

[30] B. Cao, X. Kang, J. Zhao, P. Yang, Z. Lv, and X. Liu, "Differential Evolution-based 3D Directional Wireless Sensor Network Deployment Optimization," JIOT, vol. 5, no. 5, 2018. doi: 10.1109/JIOT.2018.2801623

[31] A. Afghantoloee, S. Doodman, F. Karimipour, and M. A. Mostafavi, "Coverage Estimation of Geo-sensors in 3D Vector Environments," in GIRC, 2014. doi: 10.13140/2.1.2229.0723

[32] M. Argany, F. Karimipour, F. Mafi, and A. Afghantoloee, "Optimization of Wireless Sensor Networks Deployment Based on Probabilistic Sensing Models in a Complex Environment," JSAN, vol. 20, no. 7, 2018. doi: $10.3390 /$ jsan7020020 